\title{
An Electrophoretic Study of Proteins in Chick Embryonic Fluids ${ }^{1}$
}

\author{
SALLY E. GEELHOED AND JAMES L. CONKLIN \\ Department of Anatomy, The University of Michigan, \\ Ann Arbor, Michigan
}

\begin{abstract}
The protein fractions of 7-18 day chick serum, allantoic and amniotic fluid and egg white were demonstrated by acrylamide gel electrophoresis.

The similarity in protein fractions suggests that most of the amniotic fluid proteins are derived from egg white while serum contributes most of the allantoic fluid proteins. Several protein fractions which are unique to the respective fluids may be produced by components of the extraembryonic membranes.

The nature of the proteins found in both serum and allantoic fluid suggests a selective transfer of protein between the two fluids.
\end{abstract}

An interest in the function of the chorioallantoic membrane of the chick embryo has prompted investigations (Conklin, '66) of the membrane and the allantoic fluid. One of the unanswered questions about the allantoic fluid is the initial source of its primary components. It has been suggested (Romanoff, '60) that avian allantoic fluid is produced either by the allantoic membrane or excreted into the cloaca and thence into the allantoic lumen (op. cit.), while the amniotic fluid may originate as a transudate of serum or as a secretory product of the amnion.

An obvious way to determine whether serum might contribute to the formation of either fluid would be by comparison of the protein content of the serum and the amniotic and allantoic fluids. Strangely, however, no successful comparison of the protein components of these fluids and of embryonic serum has been made. Indeed, earlier attempts to detect similarities between the fluids and embryonic and adult serum, using paper electrophoresis, failed to demonstrate any proteins in amniotic fluid until after the thirteenth day when, following the rupture of the seroamniotic plate, the proteins of amniotic fluid were identical to ovalbumin (Marshall and Deutsch, ${ }^{5} 50$ ). These authors were able to demonstrate nine protein fractions in 13 day chick serum.

Shepard and Hottle ("49) also employed paper electrophoresis and demonstrated three protein fractions in 13 day allantoic fluid. These fractions reportedly were unlike any found in embryonic plasma.
Electrophoresis in acrylamide gel, which permits the demonstration of low concentrations of protein and exhibits great resolving power, was employed by Narayan et al. ('64) to demonstrate the serum proteins of the adult chicken.

Because of the utility and efficiency of the procedure, acrylamide gel electrophoresis was employed here to demonstrate the protein composition of chick embryonic serum, amniotic and allantoic fluid and egg white at various times during development to determine if there was any similarity in the composition of these fluids. The assumption made was that similarity in protein composition implied similarity in the origin of the protein components of the various fluids.

\section{MATERIALS AND METHODS}

Samples for electrophoresis were obtained from chick embryos of 7 to 18 days incubation age (Hamburger-Hamilton, '51). Serum was obtained by withdrawing blood from allantoic vessels with a Pasteur capillary pipette, allowing it to clot for an hour, and centrifuging to remove red blood cells and fibrin. Samples contaminated by lysed red blood cells were easily detected and eliminated as distinctive hemoglobin fractions appeared after electrophoresis and staining. While some contamination with allantoic fluid was possible, it was guarded against by using only blood withdrawn before puncture of the chorioallantoic membrane.

1 This research was supported by grant HD 00557 from the Institute of Child Health and Human Development, National Institutes of Health. 
Allantoic and amniotic fluid samples were withdrawn after puncture of the respective membranes with a Pasteur capillary pipette. Any samples contaminated by red blood cells, detectable after centrifugation, were discarded. Samples of allantoic and amniotic fluid from younger embryos often contained fractions which were too dilute to measure because of their low protein content. Therefore, some samples were concentrated by dialyzing against polyethylene glycol (Carbowax 4000), which permitted removal of water and electrolytes and concentration of high molecular weight components. Polyethylene glycol did not appear to interfere with protein components, since no qualitative differences were noted between concentrated and nonconcentrated samples.

Samples of egg white were obtained by direct puncture of the albumin sac with a Pasteur pipette prior to the twelfth day of incubation.

Electrophoresis was performed by a modification of the method of Orenstein and Davis ('61). Polyacrylamide gel (Eastman Kodak Co.) columns were prepared and allowed to polymerize, a spacer gel being added on top of the separation gel. Columns were then placed in a polystyrene electrophoretic cell suspended between two baths of $0.005 \mathrm{M}$ Tris-glycine buffer, $\mathrm{pH}$ 8.3. $1.0 \mathrm{cc}$ of $0.001 \%$ aqueous bromphenol blue added to the upper buffer served as a marker to indicate the appropriate time to terminate electrophoresis. Additionally, it was used for the calculation of $\mathrm{Rf}$ values of the protein samples,

$$
\mathbf{R f}=\begin{gathered}
\text { migration of protein fraction } \\
\text { migration of marker dye }
\end{gathered}
$$

Immediately before electrophoresis, samples were layered directly on the spacer gel, after being mixed 1:1 with a $40 \%$ sucrose solution, according to an alternative method for adding sample suggested by Davis ('64). The sucrose solution, of greater density than the buffer, lay evenly on top of the spacer gel when added carefully with a serological pipette. Ten or $20 \mu \mathrm{l}$ of the sample mixture was usually used although larger quantities $(50,100$ $\mu 1)$ of sample were tried. Sample amounts in excess of $10 \mu \mathrm{l}$ gave no better results and often, when proteins were present in high concentration, resulted in poorer resolution of individual fractions.

Electrophoresis was carried out at a constant current of $2.5 \mathrm{~mA} /$ tube, usually for 40 to 45 minutes or until the bromphenol blue marker had moved $42-45 \mathrm{~mm}$. Gel columns were then immediately removed from the tubes, simultaneously fixed and stained with a solution of $1 \%$ amido black B (E. Merck A. G.) in 7\% acetic acid for one hour. Columns were destained electrophoretically at $10 \mathrm{~mA} /$ tube using $7 \%$ acetic acid in the buffer reservoirs. After determination of the $\mathrm{Rf}$ values and photographing, columns were stored at room temperature in $7 \%$ acetic acid (Davis, '64) with no noticeable fading of protein bands occurring even after six months.

\section{RESULTS}

In analyzing the various protein fractions after electrophoresis, it seemed most meaningful to employ visual comparison of the fractions and comparison of the $\mathrm{Rf}$ values in deciding whether fractions from various fluids were the same or different.

Representative gels illustrating most, but not all, of the fractions are illustrated in figures 1-3. Examination of these figures reveals the rather precise separation and localization of the proteins after electrophoresis in acrylamide gel. This separation permitted the resolution of the total protein fractions of the fluids studied and determination of the $R f$ value for each fraction (summarized in fig. 4).

With the exception of egg white which was studied only at days 10-12, all fluids demonstrated an increase in protein fractions during development.

Serum exhibited the greatest number of fractions with thirteen fractions present by day 17. Allantoic fluid contained twelve fractions. The smallest total number of fractions, nine, was observed in amniotic Aluid.

Nine fractions were initially present in serum with one additional fraction ( $\mathrm{Rf}=$ 0.32 ) appearing on day 9 and three fractions ( $\mathrm{Rf}=0.68,0.20,0.13$ ) on day 17 . Allantoic fluid initially possessed eight fractions with additional fractions appearing on days $9(\mathrm{Rf}=0.61)$ and $14(\mathrm{Rf}=$ $0.73,0.32,0.20)$. 


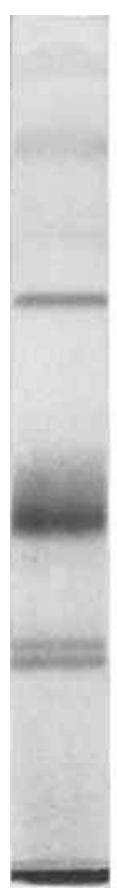

A

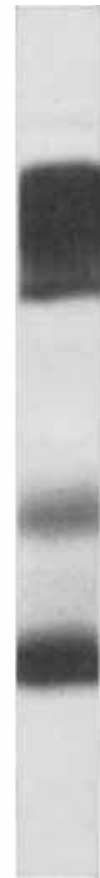

B

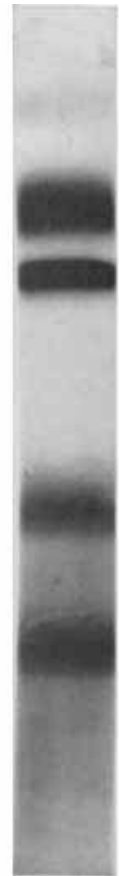

$\mathrm{C}$

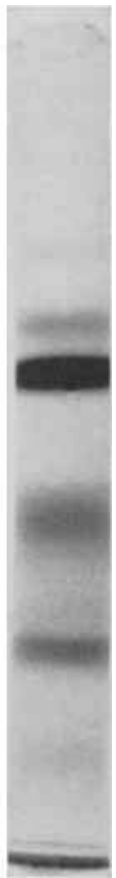

$\mathrm{D}$
Fig. 1 Acrylamide gels exhibiting protein fractions in chick embryo serum, (A) 7-8 day, (B) 9-12 days, (C) 13-16 days, (D) 17-18 days. Not all fractions are demonstrated in all columns. This is due to (1) concentration differences in various samples, (2) variation in staining, and (3) the technical difficulty in illustrating weak fractions with resultant loss of resolution of individual strong fractions. See figure 4 for identifcation of individual fractions.

There was some similarity between the protein fractions of allantoic fluid and serum. Eleven fractions were observed in both allantoic fluid and serum. However, it should be noted that only six of these were present at the seventh day. Of the remaining five fractions, four appeared in the serum at an earlier age than they were demonstrated in the allantoic fluid. The fifth fraction ( $\mathrm{Rf}=0.20$ ) appeared in the allantoic fluid on day 15 but was not observed in serum until day 17 . Only two fractions ( $R f=0.42,0.13$ ) were unique to serum while only one fraction ( $R f=$ 0.79 ) was observed in allantoic fluid and not in serum. This same fraction was also present in amniotic fluid.

Amniotic fluid exhibited a marked change in protein composition between the twelfth and thirteenth day. Prior to the

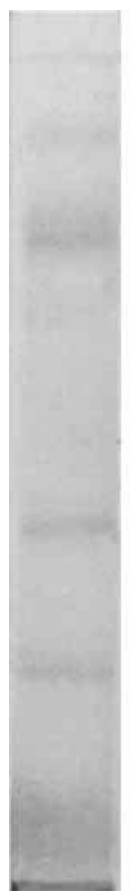

A

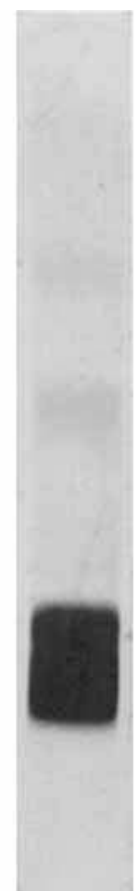

$\mathrm{B}$

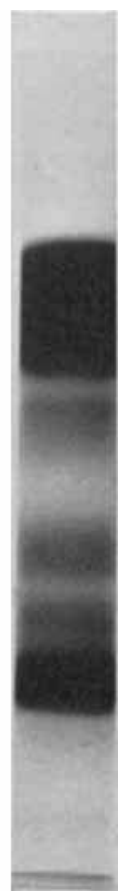

C
Fig. 2 Protein fractions of allantoic fluid, (A) 7-8 days, (B) 9-14 days, (C) 15-18 days. See figure 1 for discussion of limitations.

thirteenth day, amniotic fluid was very watery and had to be concentrated several times in order to demonstrate any protein fractions (fig. 3). However, after the twelfth day, a large quantity of protein was present in the fluid. Comparison of the fractions of 13 day amniotic fluid with egg white illustrates that most of the gained fractions were present in egg white. Although only two new fractions appeared ( $\mathrm{Rf}=0.61,0.38$ ) there was a marked increase in the concentration of all fractions, perhaps indicating that some of the original protein components of amniotic fluid and also of serum and allantoic fluid may have been derived from egg white. This is implied from the fact that all fractions of egg white were present also in amniotic and allantoic fluid and serum. A single fraction with the low $R f$ value of 0.10 was present only in amniotic fluid.

Attempts to demonstrate which of the fractions were lipo- or glycoprotein by staining of the columns were unsuccessful. However, a tentative identification was 


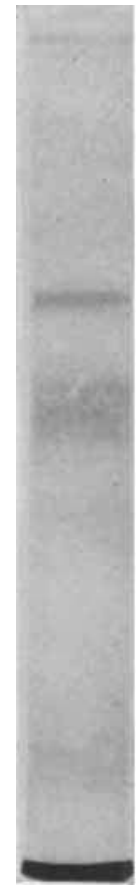

A

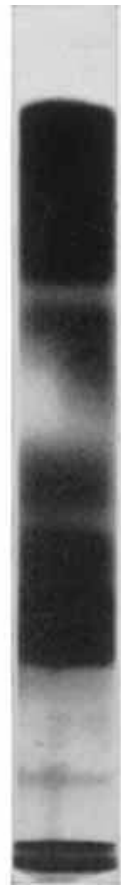

$B$

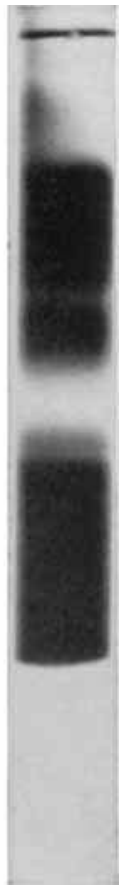

C
Fig. 3 Protein fractions of amniotic fluid (af) and egg white (ew). (A) af, 8-12 days, (B) af, 13-18 days, (C) ew, 11 days.

made by comparing the fractions with those demonstrated in human serum by Ornstein and Davis ('61) and in chicken serum by Narayan et al., ('64). The first 1 or 2 fractions ( $R f=0.92,0.84$ ) are probably prealbumins. These are followed by albumin, postalbumins, transferrins, and haptoglobins. The slower fractions ( $\mathrm{Rf}=$ $0.20,0.13$ ) are probably globulin and $\beta$ lipoprotein, in that order.

\section{DISCUSSION}

As might be predicted, all of the fluids studied, with the exception of egg white, exhibited an increase in protein components during development. The proteins acquired by the various fluids were almost without exception those with low Rf values and therefore with slow mobility in the acrylamide gel. Presumably, they are molecules of high molecular weight, although net charge is also a factor which influences electrophoretic mobility.

On the basis of $\mathrm{Rf}$ values (fig. 4) it would appear that most of the protein fractions of amniotic fluid are derived from egg white. The dramatic increase in the concentration of amniotic fluid proteins between the twelfth and thirteenth day is coincident with the rupture of the seroamniotic plate (Romanoff, '60) which admits the contents of the albumen sac to the amniotic cavity. With but a single exception, the proteins present in the amniotic thuid were also found in serum and allantoic fluid and probably have a similar origin. The one exception was a slowly migrating component ( $\mathrm{Rf}=0.10)$ which was present in amniotic fluid throughout the period studied.

From the similarity of the protein content of serum and allantoic fluid and the appearance of proteins in serum prior to their appearance in allantoic fluid it would appear that many of the protein fractions of the two fluids have a common origin. Only one fraction ( $\mathrm{Rf}=0.79$ ) was found in allantoic fluid but not in serum. This fraction was also present in amniotic fluid.

The fraction ( $\mathrm{Rf}=0.20$ ) which has an electrophoretic mobility similar to globulin (Narayan et al., '64) displayed an interesting developmental pattern. It was found initially in egg white and amniotic fluid; then appeared in allantoic fluid and finally was present in serum. It would seem that the embryo is bathed in globulin-containing amniotic fluid during development with the major portion added midway through embryogenesis via the release of the contents of the albumin sac. This globulin would be of maternal origin. Later in development embryonic synthesis of globulin begins as indicated by the appearance of this protein in the allantoic fluid and serum. The reason for the appearance of globulin in the allantoic fluid prior to its presence in serum is not clear.

While the serum proteins appear to contribute to the protein content of the allantoic fluid, the contributory process would not seem to be by way of simple diffusion. If passive diffusion were the means of transfer it would be expected that either all proteins or only those of a given size would leave the blood vessels to enter the allantoic lumen. Instead proteins of both rapid and slow electrophoretic mobility are found in both fluids while proteins of intermediate mobility are found only in serum. This would imply at least some type of 

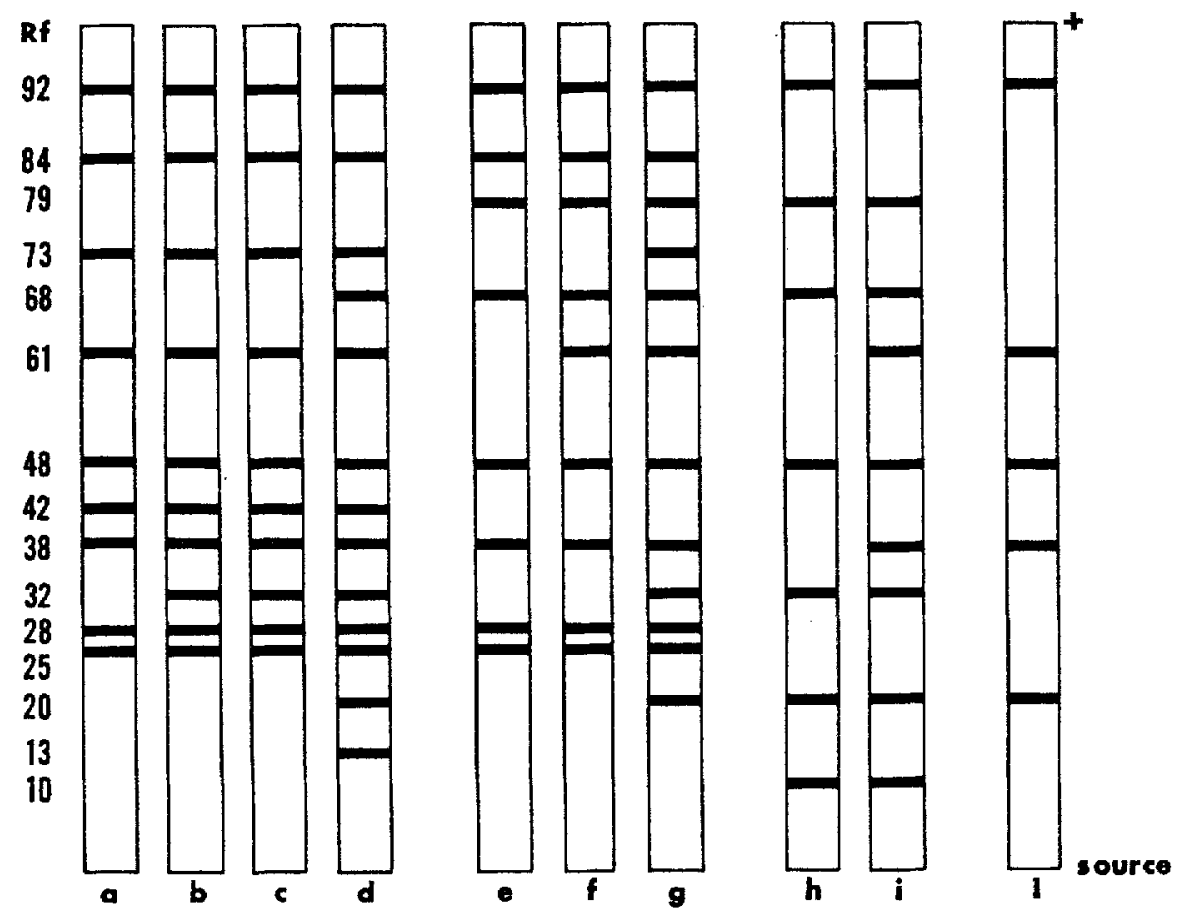

Fig. 4 Diagram illustrating total protein fractions, with $\mathrm{Rf}$ values, of chick embryonic fluids after acrylamide gel electrophoresis. (a-d) Serum, days $7-8,9-12,13-16,17-18$, respectively; (e-g) allantoic fluid, days $7-8,9-14,15-18$, respectively; (h-i) amniotic fluid, days $8-12,13-18$, respectively; (1) egg white.

selective permeability or transfer of proteins from serum to allantoic fluid. Also, it is not clear whether the passage of protein from the vascular system to the allantoic fluid occurs in the chorioallantois, the mesonephros or in some other site.

In conclusion, it seems probable that most of the proteins of the amniotic fluid are derived from the albumin sac while most of the proteins of the allantoic fluid are derived from serum. A few proteins which are unique to the extraembryonic fluids may be produced by localized secretion by components of the extraembryonic membranes. Finally, there would seem to be some selectivity in the serum proteins which pass from the plasma to the allantory fluid. The significance of this selectivity awaits more definitive identification of the various protein fractions.

\section{ACKNOWLEDGMENT}

The authors wish to acknowledge the assistance of Mrs. Constance Miller in the preparation of the manuscript.

\section{LITERATURE CITED}

Conklin, J. L. 1966 A histochemical study of mucosubstances in the chick chorioallantois. (In manuscript.)

Davis, B. J. 1964 Disc electrophoresis. II. Method and application to human serum proteins in gel electrophoresis. Annls. New York Acad. Sci., 121: 404-427.

Hamburger, V., and H. L. Hamilton 1951 A series of normal stages in the development of the chick embryo. J. Morph, 88; 49-92.

Marshall, M. E., and H. F. Deutsch 1950 Some protein changes in fluids of the developing chicken embryo. J, Biol. Chem., 185: 155-161.

Narayan, K. A., S. Narayan and F. A. Kummerow 1964 A modified disc electrophoretic method for animal blood serum proteins. J. Chromatography, 16: 187-193.

Ornstein, L., and B. J. Davis 1961 Disc electrophoresis. Distillation Products Industries, Eastman Kodak Company, Preprint.

Romanoff, A. L. 1960 The Avian Embryo, New York, The Macmillan Company, 1305 pp.

Shepard, C. C., and G. A. Hottle 1949 Studies of the composition of the livetin fraction of the yolk of hen's eggs with the use of electrophoretic analysis. J. Biol. Chem., 179: 349-357. 